\title{
Dark Matter searches with Mono-X signatures at the ATLAS experiment
}

\author{
Emma Tolley* on behalf of the ATLAS collaboration \\ Harvard University \\ E-mail: emma.tolley@cern.ch
}

At the LHC dark matter particles can be produced in association with Standard Model particles coming from initial state radiation. Searches for such processes using events with jets, photons or massive gauge bosons recoiling against large missing transverse momentum in ATLAS at $13 \mathrm{TeV}$ are presented. These "mono-X" signatures provide powerful probes to dark matter production at the LHC, allowing for the interpretation of results in terms of simplified models with pair production of WIMPs.

XXIV International Workshop on Deep-Inelastic Scattering and Related Subjects

11-15 April, 2016

DESY Hamburg, Germany

\footnotetext{
${ }^{*}$ Speaker.
} 


\section{Introduction}

Several astrophysical and cosmological anomalies observed in the universe are inconsistent with our current understanding of gravitation and particle physics [1]. The existence of a new nonluminous, non-interacting "dark matter" (DM) particle could explain these phenomena. Several theories [2] propose that dark matter will take the form of a weakly interacting massive particle (WIMP), which could couple very weakly to the Standard Model and might be produced in protonproton collisions at the LHC.

Although WIMP dark matter is invisible to the ATLAS detector, the creation of DM can be inferred when it is produced in association visible particles such as jets, photons, or electroweak bosons. This event topology is known as the "mono- $X$ " signature, and is typified by a visible particle "X" recoiling against the missing transverse momentum vector $E_{\mathrm{T}}^{\text {miss }}$. Mono-X searches look for new physics in signal regions composed of events with large missing transverse momentum, strict quality requirements on "X", and a lepton veto to reduce $W \rightarrow \ell v$ and other background processes. Background processes are constrained using control regions constructed by inverting signal region vetoes.

Mono-X search results are interpreted within a simplified model framework in which a mediator particle connects the Standard Model quarks to dark matter WIMPs [13]. The model depends on four parameters: mass of the dark matter particles $m_{\chi}$, mass of the mediator $m_{\text {med }}$, the coupling between the mediator and the quarks $g_{q}$, and the coupling between the mediator and dark matter particles $g_{\chi}$. The simplified model framework allows mono-X results to be compared with dijet resonance limits, direct and indirect dark matter detection experiments, and dark matter relic density measurements. Additionally, several searches use an effective field theory (EFT) framework to interpret results. Limits presented in the context of an EFT use the truncation method [14] to reject simulated events in which the momentum transfer is greater than the mediator mass.

This note presents a summary of dark matter searches in mono-X states with the ATLAS [3] experiment with proton-proton center-of-mass energies of $13 \mathrm{TeV}$ in data corresponding to an integrated luminosity of $3.2 \mathrm{fb}^{-1}$. Results are interpreted within the frameworks of simplified models and EFTs, and the complementary of mono-X search limits with dijet resonance constraints and dark matter direct detection experiments are shown.

\section{ATLAS Mono-X Searches and Results}

\subsection{Mono-Jet}

The mono-jet search looks for dark matter production in association with jets from initial state radiation [4]. The signal region is composed of events with $E_{\mathrm{T}}^{\text {miss }}>250 \mathrm{GeV}$, at least one jet with transverse momentum $p_{\mathrm{T}}>250 \mathrm{GeV}$, and no electrons or muons. Electroweak bosons produced in association with jets are the primary backgrounds for this search. $Z \rightarrow v v$ is the predominant background process in the signal region, followed by $W \rightarrow \ell v$ and $Z \rightarrow \ell \ell$ in which the leptons from the boson decays are lost. The $W / Z+$ jets background is constrained using three control regions constructed to be enriched in background processes by requiring one muon, two muons, and one electron, respectively. A simultaneous fit in the signal region and control regions determine $E_{\mathrm{T}}^{\text {miss }}$ dependent normalization factors to rescale the $W / Z+$ jets Monte Carlo predictions and reduce the 

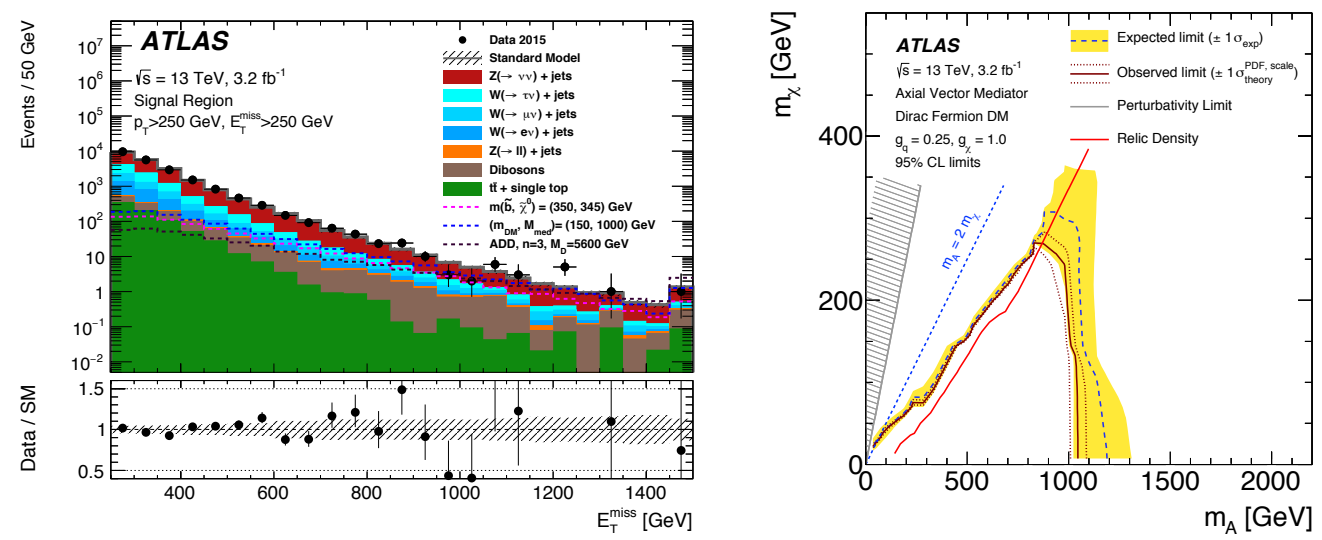

Figure 1: (Right) The $E_{\mathrm{T}}^{\text {miss }}$ distribution of events in the mono-jet signal region after the background fit. (Left) Limits as a function of dark matter mass $m_{\chi}$ and mediator mass $m_{\mathrm{A}}$ in the simplified model framework with an axial vector mediator. Dark matter is excluded up to $250 \mathrm{GeV}$ for $1 \mathrm{TeV}$ mediator. The red line shows where models in phase space predict a dark matter relic density $\Omega$ that matches $\Omega=0.12$ measured by the Planck Collaboration [17].

sensitivity to boson $p_{\mathrm{T}}$ modeling. The $W \rightarrow \mu v$ boson $p_{\mathrm{T}}$ distribution in the one-muon control region is used to model the $Z \rightarrow v v p_{\mathrm{T}}^{\text {miss }}$ distribution in the signal region. Other backgrounds yields are estimated with data-driven techniques or based on Monte Carlo simulations. 21447 signal events are observed with $21730 \pm 940$ expected background events. As no significant excess is found in the signal region, limits are calculated on the dark matter production cross section. Limits interpreted in the simplified model framework with an axial vector mediator and fixed values of $g_{q}=0.25$ and $g_{\chi}=1$ are shown in Figure 1 .

\subsection{Mono-Photon}

The mono-photon search looks for dark matter production in association with a photon from initial state radiation or a DM-photon interaction [5]. This search looks for DM in events with $E_{\mathrm{T}}^{\text {miss }}>150 \mathrm{GeV}$, one photon with transverse momentum $p_{\mathrm{T}}>150 \mathrm{GeV}$, and no electrons or muons. The $W \gamma, Z \gamma$, and $\gamma+$ jets backgrounds are constrained using three control regions, each enriched in a respective background. The normalization of these backgrounds are obtained via a simultaneous likelihood fit to the observed yields in the control regions. Contributions from processes in which an electron is misidentified as a photon are estimated by scaling yields from a sample of $e+E_{\mathrm{T}}^{\text {miss }}$ events with an electron-to-photon misidentification probability factor measured as the ratio of $e \gamma$ to $e^{+} e^{-}$events in a $Z$-boson mass window. Background contributions from events in which a jet is misidentified as a photon are estimated using a sideband counting method [7]. Limits are interpreted in the simplified model framework with an axial vector mediator and fixed values of $g_{q}$ and $g_{\chi}$. Additional limits are placed on $M$ for a dimension-7 operator EFT with a $\gamma \gamma \chi \chi$ interaction [6] as a function of dark matter mass $m_{\chi}$. Limits are shown in Figure 2. 

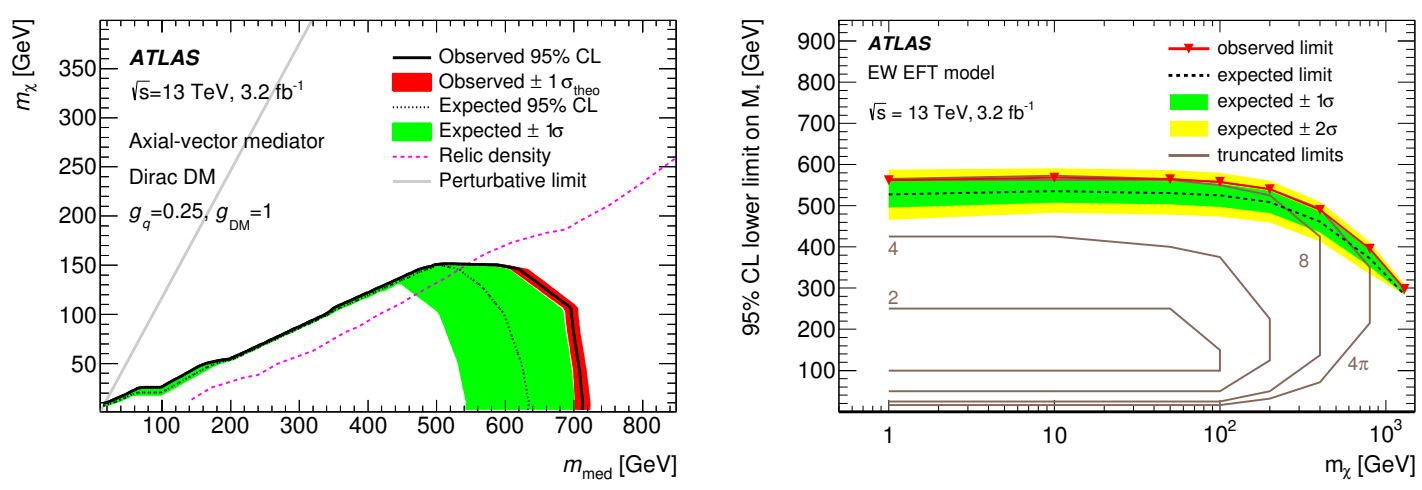

Figure 2: (Left) Limits as a function of dark matter mass $m_{\chi}$ and mediator mass $m_{\text {med }}$ in the simplified model framework with an axial vector mediator. Dark matter is excluded up to $150 \mathrm{GeV}$ for a $500 \mathrm{GeV}$ mediator. (Right) Limits on the scale of new physics $M *$ interpreted in an EFT with a $\gamma \gamma \chi \chi$ effective vertex [6]. DM mass is excluded up to $200 \mathrm{GeV}$ for $M *$ below $550 \mathrm{GeV}$.

\subsection{Mono-W/Z}

The Mono-W/Z analysis searches for dark matter production in association with a hadronically decaying $\mathrm{W}$ or $\mathrm{Z}$ boson from initial state radiation or a DM-boson interaction [8]. The hadronic W/Z decay manifests in the detector as a single large-radius jet, and can be identified as a boson decay using jet mass and substructure [9]. This search looks for DM in events with $E_{\mathrm{T}}^{\text {miss }}>250$ $\mathrm{GeV}$ and one large-radius jet. The background contributions from $Z+$ jets, $W+$ jets, and top-quark processes are constrained in dedicated control regions. Normalization factors are determined with a simultaneous fit. Limits on the signal strength $\mu=\sigma / \sigma_{\text {theory }}$ are presented as a function of dark matter mass $m_{\chi}$ and the mediator mass $m_{\text {med }}$ in Figure 3.
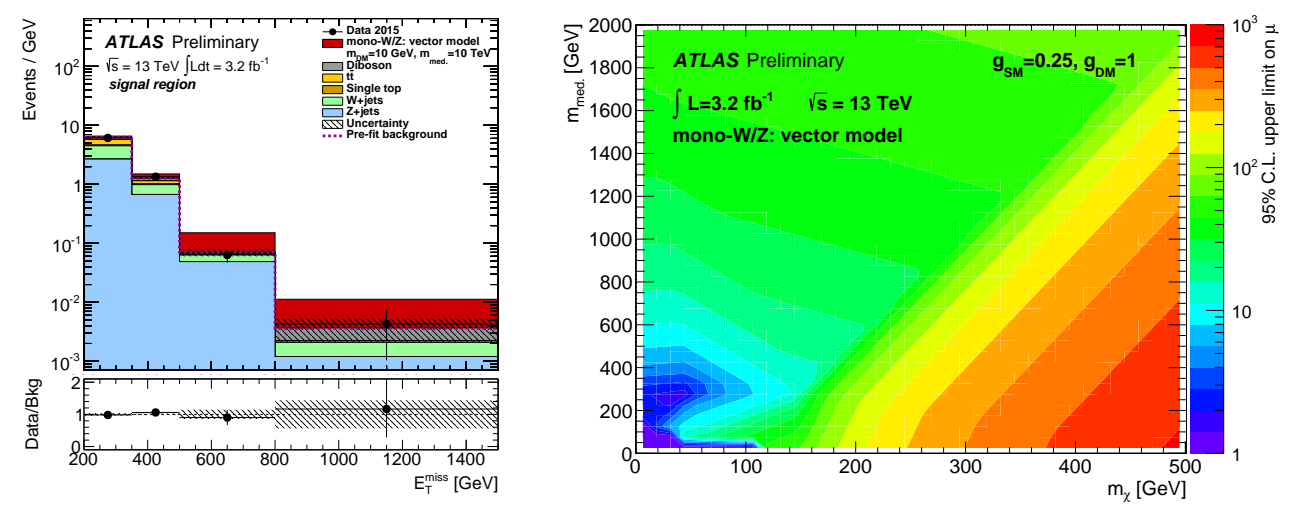

Figure 3: (Left) The $E_{\mathrm{T}}^{\text {miss }}$ distribution of events in the Mono- $W / Z$ signal region after the profilelikelihood fit [8]. (Right) Limits as a function of dark matter mass $m_{\chi}$ and mediator mass $m_{\text {med }}$ in the simplified model framework with a vector mediator. 


\subsection{Mono-Higgs}

ATLAS Mono-Higgs searches looks for dark matter production in association with a Higgs boson from a DM-Higgs interaction for three different decays of the Higgs boson: $H \rightarrow \gamma \gamma, H \rightarrow$ $b b$, and $H \rightarrow Z Z$.

The Mono- $(H \rightarrow \gamma \gamma)$ search [10] looks for DM in four signal regions with two photons with an invariant mass $m_{\gamma \gamma}$ consistent with that of the Higgs boson and with variable $E_{\mathrm{T}}^{\text {miss }}$ and $p_{\mathrm{T}}^{\gamma \gamma}$ cuts. The background is estimated with a functional fit to the $m_{\gamma \gamma}$ distribution. Limits on the signal strength $\mu=\sigma / \sigma_{\text {theory }}$ are presented as a function of mediator mass $m_{\text {med }}$ for $g_{q}=1 / 3$ and $g_{\chi}=1$ and a dark matter mass of $m_{\chi}=1 \mathrm{GeV}$ in Figure 4 (left).

The Mono- $(H \rightarrow b b)$ analysis searches for DM in two signal regions [11]. The resolved signal region has $150<\mathrm{GeV} E_{\mathrm{T}}^{\text {miss }}<500 \mathrm{GeV}$ and at least two jets. The merged signal region has $E_{\mathrm{T}}^{\text {miss }}>500 \mathrm{GeV}$ and one large-radius jet associated with two track jets. Background processes are estimated with a simultaneous shape fit to the jet mass distribution across several control regions. A one-muon control region selects for $W+$ jets and top processes, and a two-lepton control region is enriched in $Z+$ jets. The control and signal regions are further subdivided into into regions with zero, one, or two b-jets. Limits are presented as a function of dark matter mass $m_{\chi}$ and mediator mass $m_{\text {med }}$ in the simplified model framework with $g_{q}=1 / 3$ and $g_{\chi}=1$ in Figure 4 (center). The model is excluded up to $200 \mathrm{GeV}$ for a $500 \mathrm{GeV}$ mediator.

The Mono- $(H \rightarrow Z Z)$ search [12] looks for DM in events with $E_{\mathrm{T}}^{\text {miss }}>100 \mathrm{GeV}$ and four leptons consistent with the mass of the Higgs boson. The contribution from Higgs and diboson processes are taken directly from Monte Carlo simulation. The background arising from $Z+$ jets and top quark production is estimated in a simultaneous fit in dedicated control regions constructed by inverting lepton impact parameters, flavor pairing, and isolation. Limits are presented as a function of mediator mass $m_{\text {med }}$ for a dark matter mass of $m_{\chi}=1 \mathrm{GeV}$ for $g_{q}=1 / 3$ and $g_{\chi}=1$ in Figure 4(right).
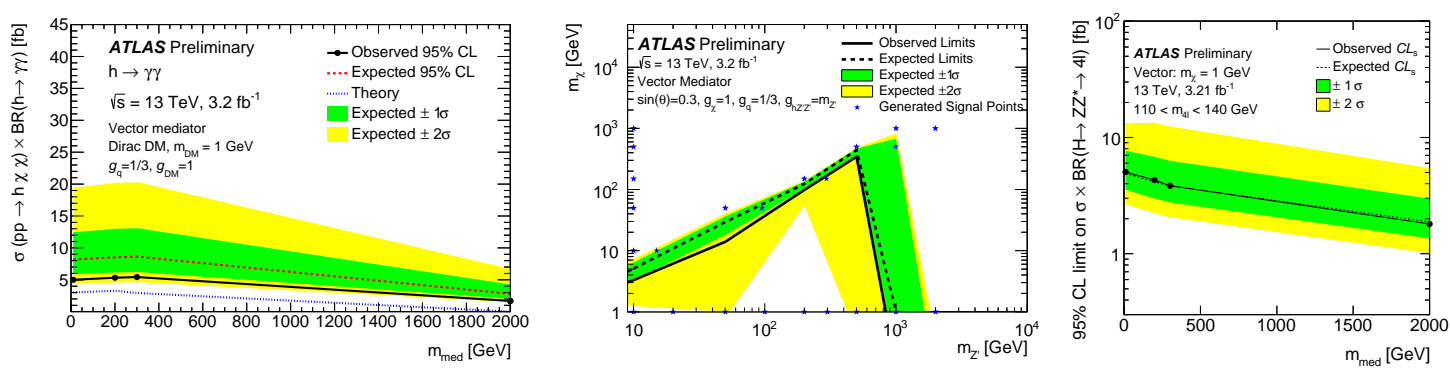

Figure 4: (Left) Mono- $(H \rightarrow \gamma \gamma)$ upper limits on the cross section as a function of the mediator mass for the vector mediator model corresponding to a dark matter mass of $1 \mathrm{GeV}$ [10]. (Center) Mono$(H \rightarrow b b)$ limits as a function of dark matter mass $m_{\chi}$ and mediator mass $m_{\text {med }}$ in the simplified model framework with a vector mediator [11]. (Right) Mono- $(H \rightarrow Z Z)$ upper limits on the cross section as a function of the mediator mass for the vector mediator model corresponding to a dark matter mass of $1 \mathrm{GeV}$ [12]. 


\section{Reinterpretation of Mono-X Limits}

Direct detection experiments set a limit on the rate of interactions between the local dark matter halo and atomic nuclei [13]. ATLAS limits can be reinterpreted using the simplified model framework and compared with direct detection limits as shown in Figure 5. LHC limits provide complementary exclusion of DM models at low values of $m_{\chi}$.
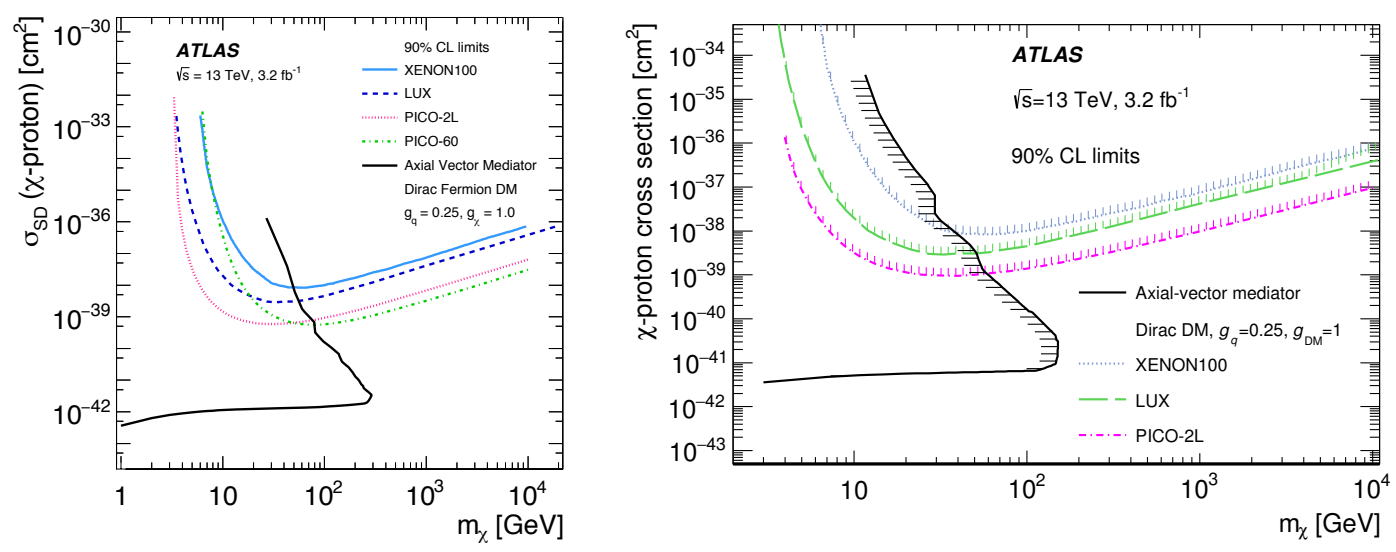

Figure 5: ATLAS mono-jet [4] (left) and mono-photon [5] (right) limits reinterpreted as limit on DM-proton scattering cross-section. The collider limits have complimentary with direct detection experiments at low dark matter mass.

Bounds on dijet resonances also constrain dark matter simplified models [15] by putting limits on the mediator decay rate to quarks. The exclusion power of mono- $X$ and dijet searches depends on the coupling values for the simplified model, as the mono-X rate scales as $g_{q}^{2} g_{\chi}^{2}$ whereas the dijet rate scales as $g_{q}^{4}$. The limits on dark matter simplified models for $g_{q}=0.25$ and $g_{\chi}=1$ from the ATLAS mono-photon, mono-jet, and dijet searches are shown in Figure 6.

\section{Conclusions}

ATLAS has searched for dark matter via the recoil of photons, jets, electroweak bosons, and the Higgs boson. As no evidence for dark matter has been observed, the results are presented as excluded regions in the framework of simplified models and effective operators. Limits are compared with dijet resonance constraints and translated into WIMP-nucleon scattering cross sections. Mono-X searches provide complimentary exclusions with searches by dedicated dark matter experiments at low dark matter mass.

\section{References}

[1] G. Bertone, D. Hooper, J. Silk, Phys. Rept. 405 (2005) 279, [arXiv:hep-ph/0404175]

[2] G. Jungman, M. Kamionkowski and K. Griest, Phys. Rept. 267 (1996) 195, [arXiv:hep-ph/9506380]

[3] ATLAS Collaboration, JINST 3 (2008) S08003.

[4] ATLAS Collaboration, submitted to Phys. Rev. D, [arXiv:1604.07773 [hep-ex]] 


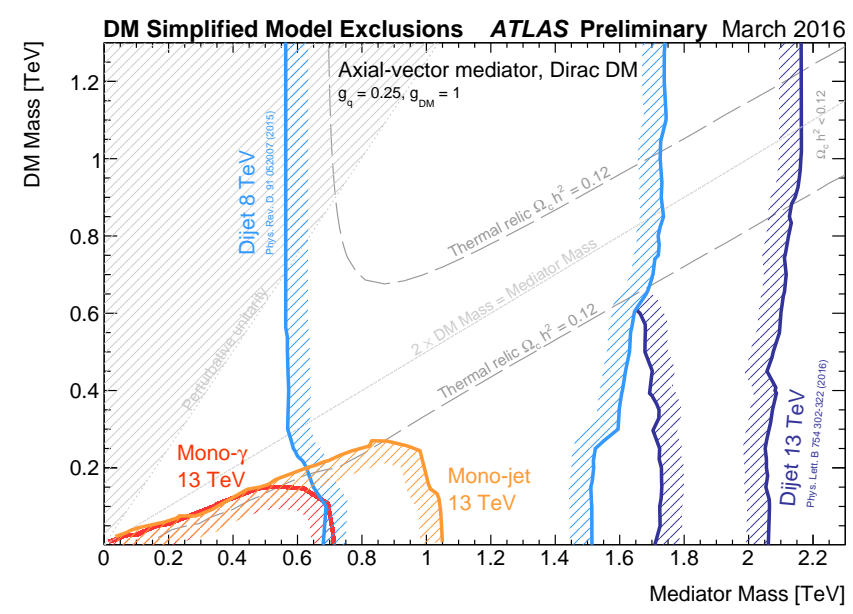

Figure 6: ATLAS mono-jet, mono-photon, and dijet limits as a function of dark matter mass and mediator mass in the simplified model framework with an axial vector mediator [16].

[5] ATLAS Collaboration, JHEP06 (2016) 059, [arXiv:1604.01306 [hep-ex]]

[6] D. Abercrombie et al., [arXiv:1507.00966 [hep-ex]]

[7] ATLAS Collaboration, Phys. Rev. D 83 (2011) 052005, [arXiv:1012.04389 [hep-hp]]

[8] ATLAS Collaboration, ATLAS-CONF-2015-080, cds.cern.ch/record/2114852

[9] ATLAS Collaboration, ATL-PHYS-PUB-2015-033, cds.cern.ch/record/2041461

[10] ATLAS Collaboration, ATLAS-CONF-2016-011, cds.cern.ch/record/2139812

[11] ATLAS Collaboration, ATLAS-CONF-2016-019, cds.cern.ch/record/2142777

[12] ATLAS Collaboration, ATLAS-CONF-2015-059, cds.cern.ch/record/2114825

[13] J. Abdallah et al., Phys. Dark Univ. 9-10 (2015) 8-23, [ arXiv:1506.03116 [hep-ph]]

[14] G. Busoni, A. De Simone, E. Morgante, and A. Riotto, Phys. Lett. B 728, 412 (2014), [arXiv:1307.2253 [hep-ph]]

[15] M. Chala et al., JHEP 1507 (2015) 089, [arXiv:1503.05916 [hep-ph]]

[16] ATLAS Collaboration, atlas.web.cern.ch/Atlas/GROUPS/PHYSICS/CombinedSummaryPlots/EXOTICS/index.html

[17] P. A. R. Ade et al. [Planck Collaboration], Astron. Astrophys. 566 (2014) A54, [arXiv:1311.1657 [astro-ph.CO]] 